\title{
COPD prevalence is increased in lung cancer, independent of age, sex and smoking history
}

\author{
R.P. Young*, R.J. Hopkins*, T. Christmas”, P.N. Black , P. Metcalf ${ }^{+}$and G.D. Gamble*
}

ABSTRACT: Chronic obstructive pulmonary disease (COPD) is a common comorbid disease in lung cancer, estimated to affect $40-70 \%$ of lung cancer patients, depending on diagnostic criteria. As smoking exposure is found in $85-90 \%$ of those diagnosed with either COPD or lung cancer, coexisting disease could merely reflect a shared smoking exposure. Potential confounding by age, sex and pack-yr smoking history, and/or by the possible effects of lung cancer on spirometry, may result in over-diagnosis of COPD prevalence.

In the present study, the prevalence of COPD (pre-bronchodilator Global Initiative for Chronic Obstructive Lung Disease $2+$ criteria) in patients diagnosed with lung cancer was $50 \%$ compared with $8 \%$ in a randomly recruited community control group, matched for age, sex and pack-yr smoking exposure $(n=602$, odds ratio $11.6 ; p<0.0001)$.

In a subgroup analysis of those with lung cancer and lung function measured prior to the diagnosis of lung cancer $(n=127)$, we found a nonsignificant increase in COPD prevalence following diagnosis (56-61\%; $p=0.45$ ). After controlling for important variables, the prevalence of COPD in newly diagnosed lung cancer cases was six-fold greater than in matched smokers; this is much greater than previously reported.

We conclude that COPD is both a common and important independent risk factor for lung cancer.

KEYWORDS: Chronic obstructive pulmonary disease, epidemiology, lung cancer, risk, spirometry

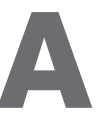
s only $10-15 \%$ of chronic smokers get lung cancer [1], host susceptibility factors have been implicated. Age, smoking history, family history and impaired lung function have been identified as key risk factors [2]. The question that then arises is: does the association between chronic obstructive pulmonary disease (COPD) and lung cancer come down to more than a shared smoking history?

Cross-sectional studies show that the prevalence of COPD is $40-70 \%$ of those diagnosed with lung cancer [3, 4], although prevalence is highly dependent on diagnostic criteria, age, sex and smoking exposure [5]. As none of these studies compared the prevalence of COPD in their lung cancer cohorts with a smoking cohort matched for these variables, the significance of this finding is uncertain. Moreover, none of these studies considered that lung cancer may itself cause an obstructive effect on spirometry. It is possible that potential confounding by age, sex and packyr smoking history on COPD prevalence, and/or the possible effects of lung cancer per se on spirometry, could result in over-diagnosis of COPD and a falsely increased association between COPD and lung cancer.

An alternative explanation is that COPD is independently and closely related to lung cancer [6] and that these diseases even share underlying host susceptibility factors $[7,8]$. This is clinically important for three reasons. First, an exaggerated or maladaptive response to smoking (or other aero-pollutant), i.e. induced airway inflammation, could be the basis of this susceptibility [8] and the target for future preventive drug therapies [9]. Evidence to support this proposition comes from recently reported genetic studies showing that both COPD [10] and lung cancer $[11,12]$ were associated with a genetic variant in the $\alpha 5$ subunit of the nicotinic acetylcholine receptor gene, previously implicated in smokinginduced lung inflammation [9]. Secondly, risk assessment tools designed to identify those at the greatest risk of lung cancer who may benefit from preventive strategies may incorporate these genetic variants, along with a history of COPD

\section{AFFILIATIONS}

*Depts of Medicine,

"Pharmacology and Clinical

Pharmacology, and

+Statistics, University of Auckland and

\#Dept of Respiratory Services, Auckland City Hospital, Auckland, New Zealand

CORRESPONDENCE

R.P. Young

Dept of Medicine

Auckland Hospital

Private Bag 92019

Auckland

New Zealand

E-mail: roberty@adhb.govt.nz

Received:

Sept 212008

Accepted after revision:

Jan 102009

First published online:

Feb 052009 
[13]. Thirdly, the diagnosis of COPD should alert patients to their elevated risk of lung cancer [14], much as elevated blood pressure does for the risk of stroke. This increased risk is independent of smoking status [15] and may have utility in prompting high-risk people to present early with new symptoms suggestive of lung cancer [16] or be a selected group for future lung cancer screening programmes. With these observations in mind, we undertook a simple crosssectional study to ascertain the prevalence of COPD in recently diagnosed lung cancer cases, to determine the effect of the cancer on spirometry and to establish to what degree (if any) COPD is found more often in lung cancer cases compared with an appropriately matched control group randomly recruited from the community.

\section{MATERIALS AND METHODS}

\section{Study subjects}

Patients with lung cancer $(n=446)$ were consecutively recruited between 2004 and 2007 following referral to a specialist lung cancer clinic at a local tertiary hospital (Green Lane Clinical Centre, Auckland, New Zealand). These patients were $>40$ yrs of age, of Caucasian ancestry (all four grandparents of Caucasian descent) and the diagnosis was confirmed through histological or cytological specimens in $95 \%$ of cases. Nonsmokers with lung cancer were excluded from this study and only those cases of primary lung cancer with the following pathological diagnosis were included: adenocarcinoma, squamous cell cancer, small cell cancer and nonsmall cell cancer (generally large cell or bronchoalveolar subtypes). Spirometry in the lung cancer cases was performed using American Thoracic Society (ATS) criteria within 3 months of lung cancer diagnosis, prior to surgery and in the absence of pleural effusions or lung collapse (partial or complete) on plain chest radiographs. Spirometry was performed after withholding short- and long-acting bronchodilators for a minimum 4 and 12 h, respectively. Among the lung cancer cases, we identified those with previous lung function testing, which was carried out on average 2 yrs prior to diagnosis (range 1-5 yrs). This was performed by the hospital lung function laboratory using ATS criteria. In a subgroup that underwent surgery for their lung cancer, we obtained lung function $\geqslant 6$ weeks after lobectomy. Control subjects were recruited from the same city suburbs from which the lung cancer cases came, during the years 2002-2005. Subjects were recruited through a random sample from the Auckland electoral rolls (response rate of 60\%) [17]. Subjects completed an investigatoradministered questionnaire that covered details of ethnicity, smoking history and previous medical history. We selected those respondents between the ages of 40 and 75 yrs, with selfdeclared European ancestry and a minimum 10 pack-yr smoking history $(n=654)$. Matching of the lung cancer cases with controls from the community-based survey was done by our biostatistician (G.D. Gamble) using the following parameters, matching one for one for each of the following: age at recruitment within 5 yrs, smoking history at recruitment within 5 pack-yrs and matching of sex. All participants gave written informed consent and the study was approved by the local ethics committee (Auckland Ethics Committee, Auckland, New Zealand). We used pre-bronchodilator spirometry and subjects were classified as having COPD according to Global
Initiative for Chronic Obstructive Lung Disease (GOLD) criteria 2 or more $[5,18]$.

\section{Statistical analysis}

Patient characteristics in the cases and controls were compared by unpaired t-tests for continuous variables and a Chi-squared test for discrete variables (Mantel-Haenszel odds ratio).

\section{RESULTS}

Table 1 summarises the clinical characteristics of our unmatched and matched lung cancer and community-based smoking controls. From a total cohort of 654 community-based randomly selected smokers aged 40-75 yrs, we identified a subgroup of 301 subjects that were closely matched one for one with the lung cancer cases. From the tertiary hospital clinic, we identified 446 lung cancer cases of Caucasian ancestry. For the community-based smoking controls, apart from age and packyr smoking history (where the total group (unmatched $n=654$ ) is younger and smoked less than the matched subgroup $(n=301))$, the smoking control subgroup is very similar in baseline characteristics to the total group. Similarly for the lung cancer cases, apart from age and pack-yr smoking history (where the total (unmatched) group is older and smoked more than the matched subgroup), the lung cancer subgroup $(n=301)$ is very similar in baseline characteristics to the total group $(n=446)$ recruited from the clinic. In the matched comparison, weight was higher among controls $(p<0.001)$ and current smoking less among controls $(p<0.001)$ when compared with lung cancer cases (table 1). In contrast, lung function and prevalence of COPD were significantly different in the matched comparison (table 1). The demographic variables, staging and histological subtypes of the lung cancer cases in this study (tables 1 and 2) are comparable to a large series published from a cohort in the USA [19], suggesting that our lung cancer cohort is representative (histology: 17\% small cell, $10 \%$ nonsmall cell, $43 \%$ adenocarcinoma, $24 \%$ squamous cell and 5\% unknown histology; staging: $29 \%$ stage 1, $10 \%$ stage 2, 31\% stage 3 and $30 \%$ stage 4 ).

On comparing lung function (table 1), we found that the lung cancer cases had consistently greater airflow limitation, regardless of COPD severity, than the matched communitybased smokers. Specifically, the forced expiratory volume in $1 \mathrm{~s}$ (FEV1), FEV1\% predicted and FEV1/forced vital capacity (FVC) ratio were lower in the lung cancer cases compared with controls. More importantly, the prevalence of COPD (prebronchodilator GOLD 2+ criteria) was 50\% in the matched lung cancer cases compared with $8 \%$ in the matched smoking controls $(n=602$; OR 11.6; $p<0.0001)$ corresponding to a sixfold greater prevalence. This prevalence is only slightly different to that seen in the unmatched cohorts. Figure 1 shows the distribution of FEV1 \% pred in our lung cancer cases $(n=301)$ compared with control smokers in a local population matched for age, ethnicity and smoking exposure $(n=301)$. Figure 2 shows the estimated proportion of lung cancer cases from smokers with COPD compared with those with normal or near normal lung function based on a GOLD 2+ prevalence of $50 \%$ among those diagnosed with lung cancer. GOLD 2+ criteria was chosen to define COPD to 1) minimise potential over-diagnosis of COPD in these older cohorts (mean age $64-65 \mathrm{yrs}$ ), in which low FEV1/FVC ratio (i.e. GOLD 1+) is 


\begin{tabular}{|c|c|c|c|c|c|}
\hline Subjects $\mathrm{n}$ & 654 & 446 & 301 & 301 & \\
\hline Males \% & 57 & 53 & 53 & 53 & \\
\hline Weight kg & $80 \pm 16$ & $69 \pm 15$ & $78 \pm 15$ & $71 \pm 16$ & $<0.001$ \\
\hline \multicolumn{6}{|l|}{ Smoking history } \\
\hline Age started smoking yrs & $18 \pm 4$ & $17 \pm 4$ & $18 \pm 4$ & $18 \pm 4$ & 0.62 \\
\hline Cigarettes $\cdot$ day $^{-1}$ & $17 \pm 9$ & $20 \pm 10$ & $20 \pm 7$ & $19 \pm 9$ & 0.33 \\
\hline Current smokers \% & 24 & 35 & 22 & 39 & $<0.001$ \\
\hline \multicolumn{6}{|l|}{ Lung function } \\
\hline $\mathrm{FEV}_{1} / \mathrm{FVC} \%$ & $81 \pm 9$ & $64 \pm 13$ & $80 \pm 10$ & $64 \pm 13$ & $<0.001$ \\
\hline \multicolumn{6}{|l|}{ Prevalence of COPD \% } \\
\hline GOLD 1+ & 10 & 60 & 15 & 65 & $<0.001$ \\
\hline GOLD 2+ & 6 & 51 & 8 & 50 & $<0.001$ \\
\hline GOLD 3+ & 1.2 & 14 & 1.3 & 15 & $<0.001$ \\
\hline \multicolumn{6}{|l|}{ History of comorbidities \% } \\
\hline Chronic bronchitis & 5 & 18 & 6 & 16 & $<0.001$ \\
\hline Asthma & 12 & 12 & 11 & 13 & 0.45 \\
\hline
\end{tabular}

Data are presented as mean $\pm \mathrm{SD}$, unless otherwise stated. FEV1: forced expiratory volume in $1 \mathrm{~s}$; \% pred: \% predicted; FVC: forced vital capacity; COPD: chronic obstructive pulmonary disease; GOLD: Global Initiative for Chronic Obstructive Lung Disease. \# : comparison for matched cohorts only.

commonly seen [18], and 2) best reflect older definitions of COPD (European Respiratory Society and ATS) [5]. The prevalence of restrictive lung function (FEV1/FVC $>70 \%$ and FVC $<80 \%$ ) was comparable in the cases and controls $(\sim 12 \%)$ but may in part reflect differences in body mass index (BMI) between the cases and controls [18, 20]. No relationship with lung function (or COPD prevalence) was seen after subgrouping lung cancer cases according to staging although COPD prevalence was slightly higher in small cell and squamous cell lung cancers (table 2).

In a subgroup analysis of inoperable lung cancer cases $(n=127)$, we identified lung cancer cases who had already undergone lung function testing on average 21 months (range 1-5 yrs) prior to lung cancer diagnosis. Although spirometry was slightly reduced at the time of diagnosis (table 3 ), we found the prevalence of COPD (as defined) only increased from $56 \%$ to $61 \%(p=0.45)$. The higher frequency of COPD in this subgroup likely reflects the greater impairment of lung function and associated inoperability.

In a second subgroup analysis of operable lung cancer cases $(n=100)$, we identified lung cancer cases who had undergone lobectomy for their lung cancer and had repeat lung function testing on average 23 months (range 1-5 yrs) after surgery. This group was comparable to the larger lung cancer cohorts: $51 \%$ male, mean age 68 yrs, mean smoking history 37 pack-yrs, mean height $167 \mathrm{~cm}$ and mean weight $72 \mathrm{~kg}$. In this subgroup we found post-operative lung function was reduced (table 3) and the prevalence of COPD (as defined previously) increased from $44 \%$ to $60 \%(\mathrm{p}=0.02)$.

\section{DISCUSSION}

In the present study, we found the prevalence of COPD (prebronchodilator GOLD 2+) to be 50\% in 301 lung cancer cases and $8 \%$ in our matched sample of community-based smoking controls with no lung cancer. In a subgroup analysis of lung cancer cases, in whom spirometry had been carried out prior to and after diagnosis $(n=127)$, we found a small and nonsignificant increase in the prevalence of COPD following lung cancer diagnosis $(56 \%$ and $61 \%$, respectively; $p=0.45)$. The $8 \%$ prevalence of COPD in the community-based smoking controls reported here is consistent with recently published prevalence studies worldwide [5]. We show that the prevalence of COPD was more than six-fold greater in the lung cancer cohort compared with matched smoking controls and that this did not result from over-diagnosis. We believe this may be the first case-control study of COPD prevalence in lung cancer where controls were carefully matched and the effects of lung cancer on spirometry was examined.

A number of studies have reported the results of their spirometry in newly diagnosed lung cancer [3,4]. Although these studies use different spirometric criteria, they show that approximately $40-70 \%$ of lung cancer cases have coexisting COPD. Although our study showed a comparable COPD prevalence of $50 \%$, these are all cross-sectional studies. In a prospective study, baseline spirometry was carried out and 


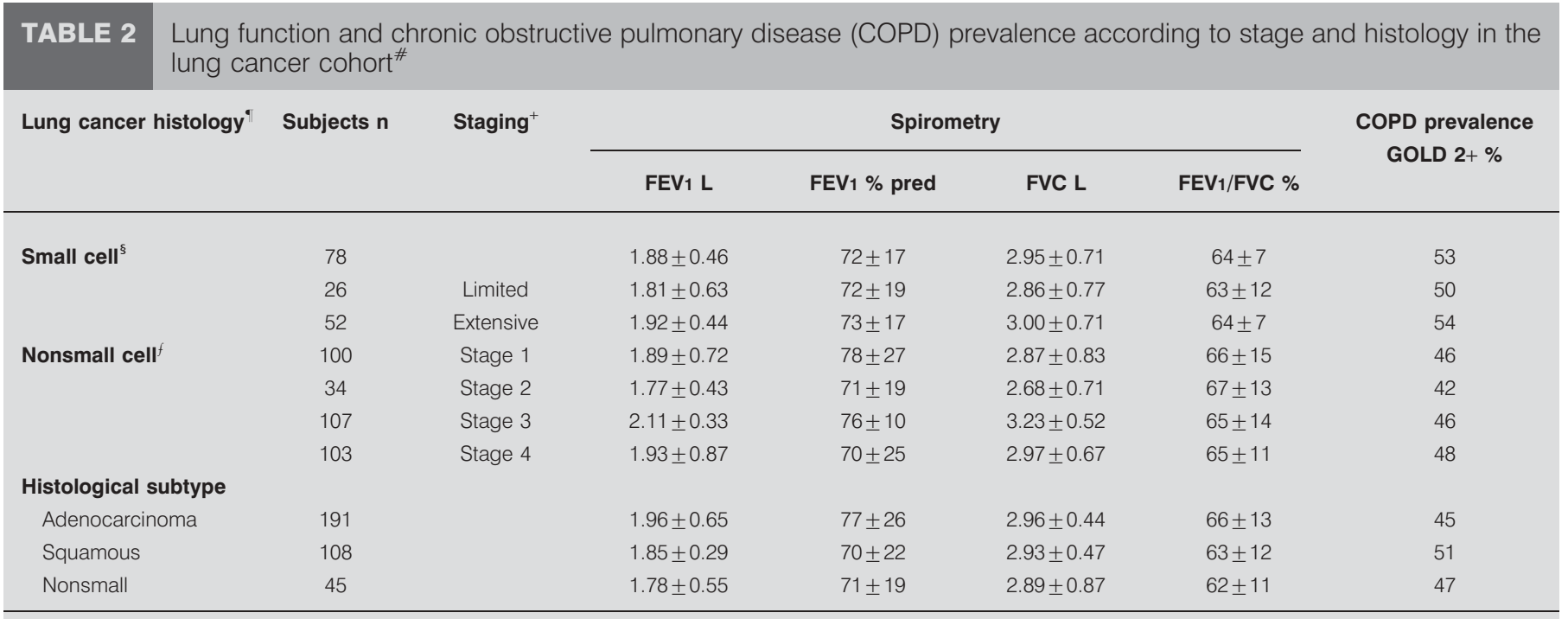

Data are presented as mean \pm SD, unless otherwise stated. FEV1: forced expiratory volume in 1 s; \% pred: \% predicted; FVC: forced vital capacity; GOLD: Global Initiative for Chronic Obstructive Lung Disease. ${ }^{\#}: \mathrm{n}=446$; ${ }^{\circ}$ : histology not available in $5 \%$ of all lung cancer cases; ${ }^{+}$: data for accurate staging was available in $85 \%$ of nonsmall cell lung cancer cases; ${ }^{\text {s: }}$ total $n=78,17 \%$; $^{\text {: total }} n=344,77 \%$.

incident lung cancer cases were identified over a 20-yr followup period [21]. In the present study, $48 \%$ of those diagnosed with lung cancer had pre-existing COPD (based on the similar spirometric criteria) on baseline spirometry. We assume that had spirometry been done closer to the time of diagnosis, the prevalence of COPD would have been somewhat higher. These findings support those of a recently reported prospective study by WILsON et al. [22], in which lung cancer $(n=99)$ was diagnosed following yearly computed tomography (CT) screening. In the WILSON et al. [22] study, the prevalence of COPD according to GOLD $1+, 2+$ and $3+$ criteria was $67 \%, 51 \%$ and $15 \%$, respectively, almost identical to those reported here. As expected, the prevalence of COPD (GOLD 2+) in that study was $29 \%$ in the nonrandomised "noncases" who were younger and had smoked less. In contrast to the current study,

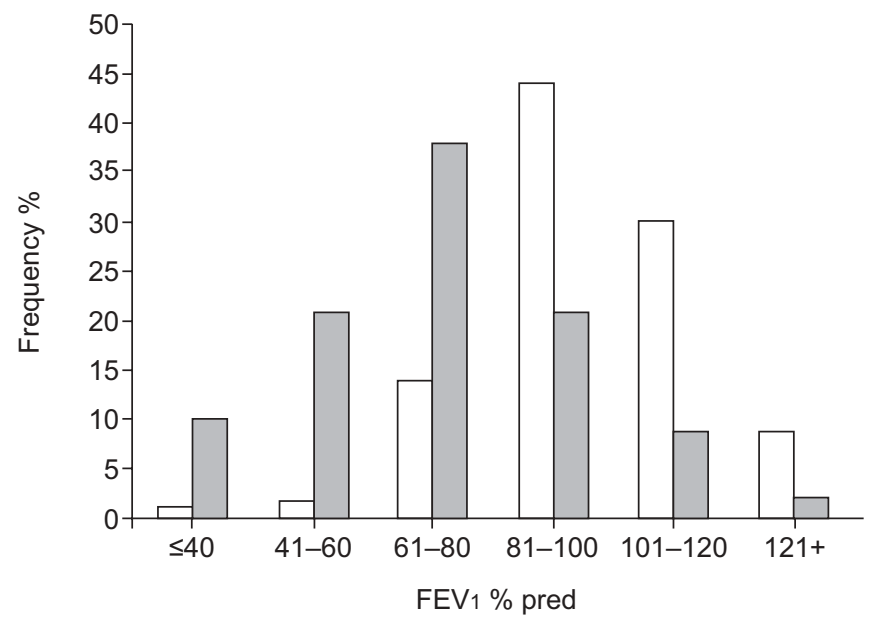

FIGURE 1. Frequency distribution of forced expiratory volume in $1 \mathrm{~s}(\mathrm{FEV} 1) \%$ predicted in smoking controls $(\square)$ and lung cancer cases $(\square)(n=602)$ matched for age, sex and smoking history. emphysema was systematically scored for severity by WILSON et al. [22] and, consistent with others, shown to be independently associated with lung cancer. We found $\sim 12 \%$ of our lung cancer cases had restrictive lung function comparable to other studies [21]. However, we did not find any difference in restrictive lung function between cases and controls, although differences in BMI may be, in part, obscuring any difference $[18,20]$.

Although the above studies are in agreement, and confirm that a half or more of lung cancer cases have coexisting COPD, it is

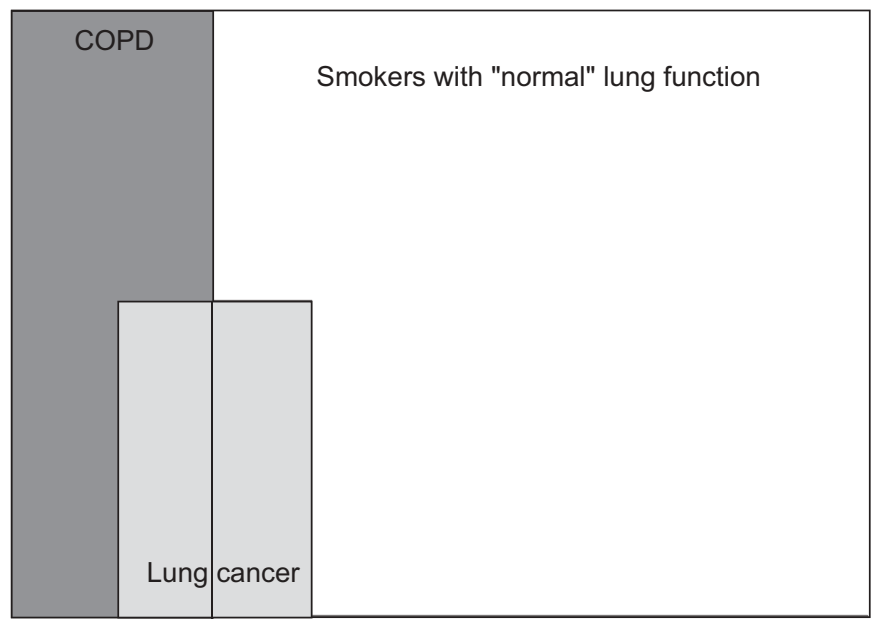

FIGURE 2. Relationship between lifetime risk of chronic obstructive pulmonary disease (COPD; Global Initiative for Chronic Obstructive Lung Disease (GOLD) 2+) and lung cancer in chronic smokers $(n=100)$. Assuming $\sim 20$ (20\%) out of 100 of smokers get COPD (GOLD 2+; $\mathbf{m}$ ) and 10 (10\%) out of 100 of smokers get lung cancer ( $\square$ ) then if $50 \%$ of the latter have pre-existing COPD then five (25\%) out of 20 with COPD get lung cancer while five (6\%) out of 80 with "normal" lung function get lung cancer. 


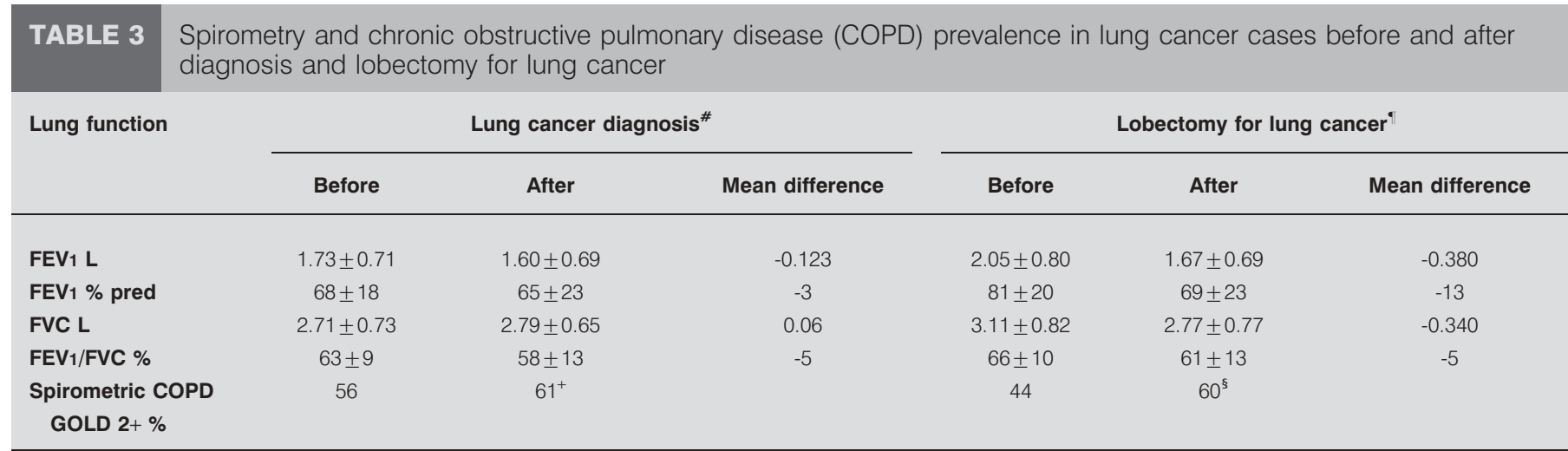

Data are presented as mean \pm SD, unless otherwise stated. FEV1: forced expiratory volume in 1 s; \% pred: \% predicted; FVC: forced vital capacity; GOLD: Global Initiative for Chronic Obstructive Lung Disease. ${ }^{\#}: n=127 ;{ }^{\top}: n=100 ;{ }^{+} p=0.45 ;{ }^{s}: p=0.02$.

not clear whether (or by how much) the presence of lung cancer may alter lung function at the time of diagnosis of lung cancer. The question then arises: Does the presence of lung cancer itself alter the spirometry and cause an over-estimate of COPD prevalence? To the best of our knowledge, no studies have attempted to assess the change in lung function before and after lung cancer diagnosis. In a subgroup analysis of lung cancer cases $(n=127)$, we have identified patients with lung function tests prior to their diagnosis of lung cancer (mean 21 months). These patients had undergone spirometry primarily for symptoms of breathlessness. In comparing lung function before and after diagnosis of lung cancer, we found only a small reduction in lung function (table 3 ) and a nonsignificant increase in COPD prevalence from $56 \%$ to $61 \%$ in this cohort $(\mathrm{p}=0.45)$. Lung function was measured on average 21 months (range 1-5 yrs) before the diagnosis of lung cancer. This observation suggests that over-diagnosis of COPD resulting from lung cancer per se is only modest relative to the prevalence observed in a matched smoking control cohort. Support for this conclusion comes from the study of WILSON et al. [22], where early stage lung cancers were diagnosed prospectively during CT screening and yet COPD prevalence at baseline was very comparable to prevalence reported in the present study (i.e. no suggestion of over-diagnosis of COPD due to the presence of more advanced stages of lung cancer itself). Further support for this comes from the observation that lung function (or COPD prevalence) was not significantly affected by lung cancer stage (table 2). In contrast, the effect of surgery to resect the tumour might alter the prevalence of COPD. Studies examining lung function after lobectomy suggest that lung function is only mildly affected [23, 24]. The results from our study are very similar to those from WIN et al. [23], who in a similarly sized study reported pre-operative FEV1 of $\sim 2 \mathrm{~L}$, dropping on average $600 \mathrm{~mL}$, compared with $400 \mathrm{~mL}$ in our study (table 3 ). Not surprisingly, this results in a significant increase in the prevalence of COPD from $44 \%$ to $60 \%(p=0.02)$ in the group who have had surgery.

The confirmation that $\sim 50 \%$ of lung cancer cases have coexisting moderate-to-severe (GOLD 2+) COPD has a number of implications. First, it suggests that a disproportionate number of lung cancer cases occur in smokers with pre-existing COPD compared with those with normal (or near normal) lung function (fig. 2). Prospective studies suggest that $20 \%$ of smokers get COPD [25] and prevalence studies suggest $\sim 10 \%$ of the smoking population, in a comparable age band to those with lung cancer (40-75 yrs), have COPD [5]. On the basis that $\sim 50 \%$ of lung cancer cases have co-existing moderate to severe COPD and, conservatively, $10 \%$ of chronic smokers get lung cancer, then a disproportionate number of lung cancer cases stem from patients with pre-existing COPD (one in four or $25 \%$ get lung cancer) compared with those smokers with "normal" lung function (one in 16 or $6 \%$ get lung cancer; fig. 2). We suggest that the risk of lung cancer among those with COPD may be closer to six-fold higher, much greater than the estimated two-fold increased risk previously associated with COPD [26]. Our results are consistent with those of prospective studies which also show, after adjustment for smoking, that COPD (based on GOLD 2+) confers up to a sixfold greater risk for lung cancer when compared with smokers with truly normal lung function [6, 21, 27]. These studies suggest that impaired lung function (based on reduced FEV1) is more important than age or smoking exposure (measured as pack-yrs) $[15,27]$. In a small CT screening study from Spain, the vast majority of lung cancer cases $(20(87 \%)$ out of 23$)$ had either spirometric evidence of COPD (16 (69\%) out of 23 with GOLD 1+) or radiological evidence of emphysema of variable severity (17 (74\%) out of 23) [28]. Furthermore, mortality studies of patients with COPD suggest $20-30 \%$ die from lung cancer [29]. Such a strong association suggests COPD should be considered the most important underlying risk factor for lung cancer, greater than that attributed to smoking dose or age. Such a view is supported by a recently published study showing that even in nonsmokers, impaired lung function is associated with an increased risk of lung cancer [15]. Collectively, these studies show that not only is COPD (or airflow limitation) closely associated with lung cancer, independent of smoking exposure dose and age, but the magnitude of the association is much greater than generally appreciated.

Certainly if obstructive pulmonary function carries up to a sixfold increase in risk for lung cancer, it is much greater than that seen for other clinical variables, such as elevated blood pressure or cholesterol (each conferring a two-fold increased risk for coronary artery disease) that are routinely measured 
for risk assessment and targeted cardiovascular prevention. This argues strongly for the routine use of spirometry in smokers to identify those with COPD and those with a significantly elevated risk for lung cancer, both of which have previously been shown to assist in smoking cessation [30-32].

A second implication from this strong association between COPD and lung cancer is the possibility that both diseases result from shared pathogenic mechanisms. It has been hypothesised that COPD is due to an inherent susceptibility (exaggerated or maladaptive response) to chronic inflammation [7-10, 14]. Interestingly, smoking-induced airway inflammation typically persists in those smokers with COPD for many years after quitting smoking [33]. This persistent inflammation may, in part, explain why $\sim 50 \%$ of lung cancer cases are found in ex-smokers $[3,10,19]$. We propose that susceptibility to lung cancer and COPD results from overlapping or shared genetic effects [7-10, 13, 14], most likely expressed through smokinginduced inflammation. Support for this hypothesis comes from recently reported genetic association studies identifying a genetic variant in the $\alpha 5$ subunit of the nicotinic acetylcholine receptor gene, which has been implicated in smoking-induced lung inflammation [9], with both COPD [10] and lung cancer $[11,12]$. Assuming this is true, and that other genetic variants confer susceptibility to both lung cancer and COPD [7], then common pathological pathways could be targeted for preventive treatment.

A third implication of the apparently close relationship between COPD and lung cancer is in the development of risk tools designed to identify those at greatest risk of lung cancer. As is the case for risk tools for other common conditions such as breast cancer (Gail Score for breast cancer) and coronary artery disease (Framingham score for myocardial infarction), increasing age is central to the assessment of the risk of lung cancer $[13,34]$. Other variables used in lung cancer risk tools include smoking history, asbestos exposure and the presence of pre-existing lung disease, notably COPD [10, 13, 34]. The results of our study and the other studies discussed above emphasise the importance of COPD as an important and independent risk variable in the risk assessment of lung cancer. We believe that, just as it is important to measure and document blood pressure (for risk of future stroke), bone mineral density (for risk of future fractures) or BMI (for risk of future diabetes), lung function should be measured and recorded for assessing the risk of future lung cancer [10, 13$15,34]$. The assessment of lung cancer risk and the utility of measuring lung function have potential clinical benefits in smoking cessation [30-32] and targeted CT screening [35]. There may also be utility in the early diagnosis of lung cancer where delays in the diagnosis [16], tumour size and mortality are closely related [36].

In summary, the close relationship between COPD and lung cancer identified in this and other studies is not just about a shared smoking exposure, but likely to reflect in part, a shared genetic susceptibility to chronic smoking-induced inflammation. This association has clinical implications for the wider use of spirometry for the early identification of those at the greatest risk of lung cancer $[10,13-15,34]$ and those who will have the most to gain from targeted smoking cessation and early diagnostic work-up for lung cancer [35].

\section{STATEMENT OF INTEREST}

None declared.

\section{REFERENCES}

1 Mattson ME, Pollack ES, Cullen JW. What are the odds that smoking will kill you? Am J Pub Health 1987; 77: 425-431.

2 Alberg AL, Brock MV, Samet JM. Epidemiology of lung cancer: looking to the future. J Clin Oncol 2005; 23: 3175-3185.

3 Loganathan RS, Stover DE, Shi W, et al. Prevalence of COPD in women compared to men around the time of diagnosis of primary lung cancer. Chest 2006; 129: 1305-1312.

4 Congleton J, Muers MF. The incidence of airflow obstruction in bronchial carcinoma, its relation to breathlessness, and response to bronchodilator therapy. Respir Med 1995; 89: 291-296.

5 Buist AS, McBurnie MA, Vollmer WM, et al. International variation in the prevalence of COPD (the BOLD study): a population-based prevalence study. Lancet 2007; 370: 741-750.

6 Skillrud DM, Offord KP, Miller RD, et al. Higher risk of lung cancer in chronic obstructive pulmonary disease: a prospective matched controlled study. Ann Intern Med 1986; 105: 503-507.

7 Schwartz AG, Ruckdeschel JC. Familial lung cancer: genetic susceptibility and relationship to chronic obstructive pulmonary disease. Am J Respir Crit Care Med 2006; 173: 16-22.

8 Brody JS, Spira A. Chronic obstructive pulmonary disease, inflammation, and lung cancer. Proc Am Thorac Soc 2006; 3: 535-538.

9 Gwilt CR, Donnelly LE, Rogers DF. The non-neuronal cholinergic system in the airways: an unappreciated regulatory role in pulmonary inflammation? Pharmacol Ther 2007; 115: 208-222.

10 Young RP, Hopkins RJ, Hay BJ, et al. Lung cancer gene associated with COPD: triple whammy or possible confounding effect? Eur Respir J 2008; 32: 1158-1164.

11 Hung RJ, McKay JD, Gaborieau V, et al. A susceptibility locus for lung cancer maps to nicotinic acetylcholine receptor subunit genes on 15q25. Nature 2008; 452: 633-637.

12 Amos CI, Wu X, Broderick P, et al. Genome-wide association scan of tag SNPs identifies a susceptibility locus for lung cancer at 15q25.1. Nat Genet 2008; 40: 1-7.

13 Young RP, Hopkins RJ, Gamble GD, et al. A gene based risk score identifies smokers and ex-smokers at high risk of lung cancer. Respirology 2008; 13: Suppl. 2, TP143.

14 Young RP, Hopkins RJ, Eaton TE. Forced expiratory volume in one second: not just a lung function test but a marker of premature death from all causes. Eur Respir J 2007; 30: 616-622.

15 Turner MC, Chen Y, Krewski D, et al. Chronic obstructive pulmonary disease is associated with lung cancer mortality in a prospective study of never smokers. Am J Respir Crit Care Med 2007; 176: 285-290.

16 Hamilton W, Peters TJ, Round A, et al. What are the clinical features of lung cancer before the diagnosis is made? A population based case-control study. Thorax 2005; 60: 1059-1065.

17 Metcalf $\mathrm{P}$, Scragg R, Schaaf D, et al. Trends in major cardiovascular risk factors in Auckland, New Zealand: 1982 to 2002-2003. NZ Med J 2006; 119: 1-16.

18 Mannino DM, Buist AS, Vollmer WM. Chronic obstructive pulmonary disease in the older adult: what defines abnormal lung function? Thorax 2007; 62: 237-241.

19 Yang P, Allen MS, Aubry MC, et al. Clinical features of 5,628 primary lung cancer patients; experience at Mayo clinic from 1997 to 2003. Chest 2005; 128: 452-462.

20 Thyagarajan B, Jacobs DR, Apostol GG, et al. Longitudinal association of body mass index with lung function: the Cardia study. Respir Res 2008; 9: 31-40. 
21 Mannino DM, Aguayo SM, Petty TL, et al. Low lung function and incident lung cancer in the United States: data from the first National Health and Nutrition Examination Survey follow-up. Arch Intern Med 2003; 163: 1475-1480.

22 Wilson DO, Weissfeld JL, Balkan A, et al. Association of radiographic emphysema and airflow obstruction with lung cancer. Am J Respir Crit Care Med 2008; 178: 738-744.

23 Win $\mathrm{T}$, Jackson A, Sharples L, et al. Relationship between pulmonary function and lung cancer surgical outcome. Eur Respir J 2005; 25: 594-599.

24 Baldi S, Ruffini E, Harari S, et al. Does lobectomy for lung cancer in patients with chronic obstructive pulmonary disease affect lung function? A multicentre national study. J Thorac Cardiovasc Surg 2005; 130: 1616-1622.

25 Lokke A, Lange P, Scharling H, et al. Developing COPD: a 25 year follow up study of the general population. Thorax 2006; 61: 935-939.

26 Wasswa-Kintu S, Gan WQ, Man SFP, et al. Relationship between reduced forced expiratory volume in one second and the risk of lung cancer: a systematic review and meta-analysis. Thorax 2005; 60: $570-575$.

27 Anthonisen NR. Prognosis in chronic obstructive pulmonary disease: results from multicenter clinical trials. Am Rev Respir Dis 1999; 140: S95-S99.
28 de Torres J, Bastarrika G, Wisnivesky JP, et al. Assessing the relationship between lung cancer risk and emphysema detected on low dose CT of the chest. Chest 2007; 132: 1932-1938.

29 The Lung Health Study Research Group. Effect of inhaled triamcinolone on the decline in pulmonary function in chronic obstructive pulmonary disease. N Engl J Med 2000; 343: 1902-1909.

30 Taylor KL, Cox LS, Zinke N, et al. Lung cancer screening as a teachable moment for smoking cessation. Lung Cancer 2007; 56: 125-134.

31 Bednarek M, Gorecka D, Wielgomas J, et al. Smokers with airway obstruction are more likely to quit smoking. Thorax 2006; 61: 869-873.

32 Parkes G, Greenhalg T, Griffin M, et al. Effect on smoking quit rate of telling patients their lung age: the Step2quit randomised controlled trial. BMJ 2008; 336: 598-600.

33 Ind PW. COPD progression and airway inflammation: uncoupled by smoking cessation. Eur Respir J 2005; 26: 764-766.

34 Spitz MR, Hong WK, Amos CI, et al. A risk model for prediction of lung cancer. J Natl Cancer Inst 2007; 99: 715-772.

35 Bechtel JJ, Kelley WA, Coons TA, et al. Lung cancer detection in patients with airflow obstruction identified in a primary care outpatient practice. Chest 2005; 127: 1140-1145.

36 Clinical tumour size and prognosis in lung cancer. Bronchogenic Carcinoma Cooperative Group of the Spanish Society of Pneumology and Thoracic Surgery. Eur Respir J 1999; 14: 812-816. 